\title{
Evaluation of the WRF-UCM mesoscale model and ECMWF global operational forecasts over the Paris region in the prospect of tracer atmospheric transport modeling
}

Jinghui $\operatorname{Lian}^{1}{ }^{*}$, Lin $\mathrm{Wu}^{2,1}$, François-Marie Bréon ${ }^{1}$, Grégoire Broquet ${ }^{1}$, Robert Vautard ${ }^{1}, \mathrm{~T}$. Scott

Zaccheo $^{3}$, Jeremy Dobler ${ }^{4}$ and Philippe Ciais ${ }^{1}$

${ }^{1}$ Laboratoire des Sciences du Climat et de l'Environnement, CEA-CNRS-UVSQ, Université

Paris-Saclay, Gif-sur-Yvette, France

${ }^{2}$ State Key Laboratory of Atmospheric Boundary Layer Physics and Atmospheric Chemistry,

Institute of Atmospheric Physics, Chinese Academy of Sciences, Beijing, China

${ }^{3}$ Atmospheric and Environmental Research, Lexington, Massachusetts, United States

${ }^{4}$ Harris Corporation, Fort Wayne, Indiana, United States

*Jinghui.Lian@1sce.ipsl.fr

\section{Supplemental Files}

Table S1. Statistics for wind with wind speeds higher than or equal to $3 \mathrm{~m} / \mathrm{s}$ considering all weather stations.

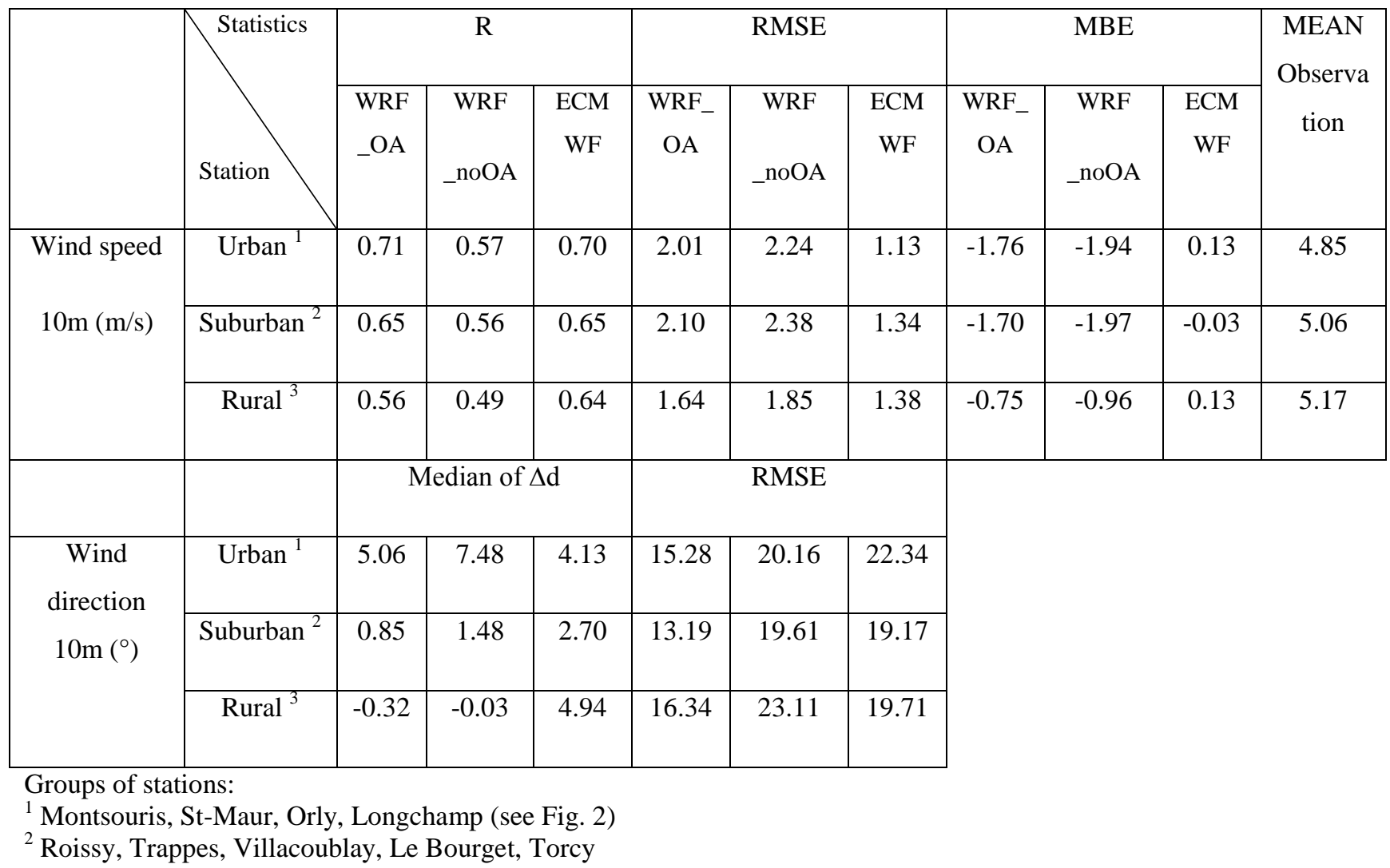


${ }^{3}$ La Brosse, Melun, Toussus, Dourdan, Changis, Orgerus, Nemours, Chevru, Nangis

Table S2. Statistics for surface temperature and wind from December 2015 to November 2016

(a) Correlation coefficient

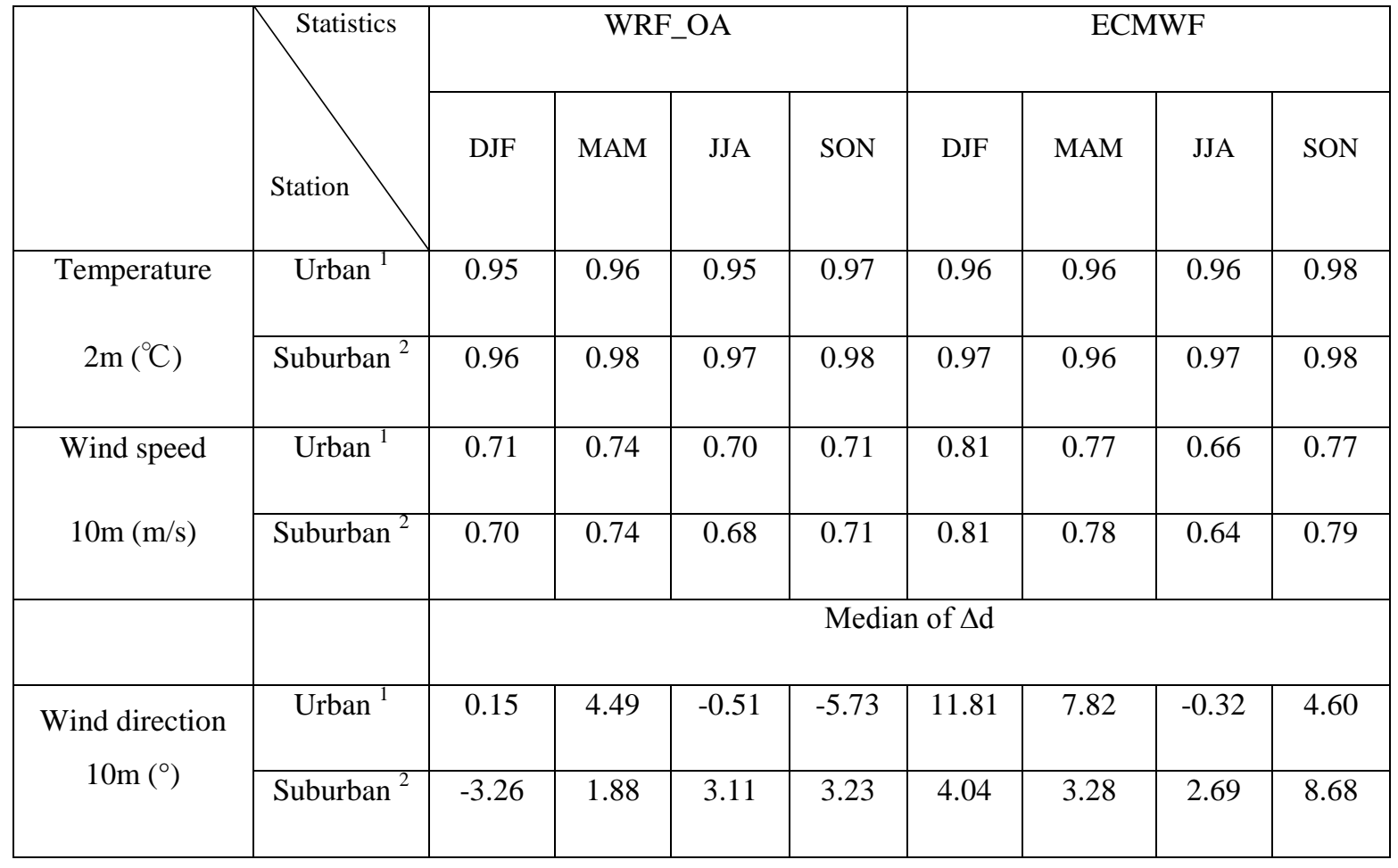

(b) Root-mean-square error

\begin{tabular}{|c|c|c|c|c|c|c|c|c|c|}
\hline & $\mathrm{Sta}$ & \multicolumn{4}{|c|}{ WRF_OA } & \multicolumn{4}{|c|}{ ECMWF } \\
\hline & & DJF & MAM & JJA & SON & DJF & MAM & JJA & SON \\
\hline Temperature & Urban $^{1}$ & 1.44 & 1.46 & 1.60 & 1.61 & 1.31 & 1.76 & 1.62 & 1.36 \\
\hline $2 \mathrm{~m}\left({ }^{\circ} \mathrm{C}\right)$ & Suburban $^{2}$ & 1.30 & 1.18 & 1.19 & 1.23 & 1.07 & 1.42 & 1.28 & 1.14 \\
\hline Wind speed & Urban $^{1}$ & 1.44 & 1.41 & 1.24 & 1.23 & 1.45 & 1.23 & 1.03 & 1.23 \\
\hline $10 \mathrm{~m}(\mathrm{~m} / \mathrm{s})$ & Suburban $^{2}$ & 1.73 & 1.48 & 1.33 & 1.39 & 1.29 & 1.23 & 1.17 & 1.18 \\
\hline
\end{tabular}




\begin{tabular}{|c|c|c|c|c|c|c|c|c|c|}
\hline $\begin{array}{c}\text { Wind direction } \\
10 \mathrm{~m}\left({ }^{\circ}\right)\end{array}$ & Urban $^{1}$ & 32.8 & 41.4 & 42.3 & 50.7 & 32.1 & 45.5 & 59.5 & 51.2 \\
\cline { 2 - 9 } & Suburban $^{2}$ & 27.5 & 37.2 & 40.3 & 42.6 & 26.2 & 43.2 & 58.3 & 47.0 \\
\hline
\end{tabular}

(c) Mean bias error

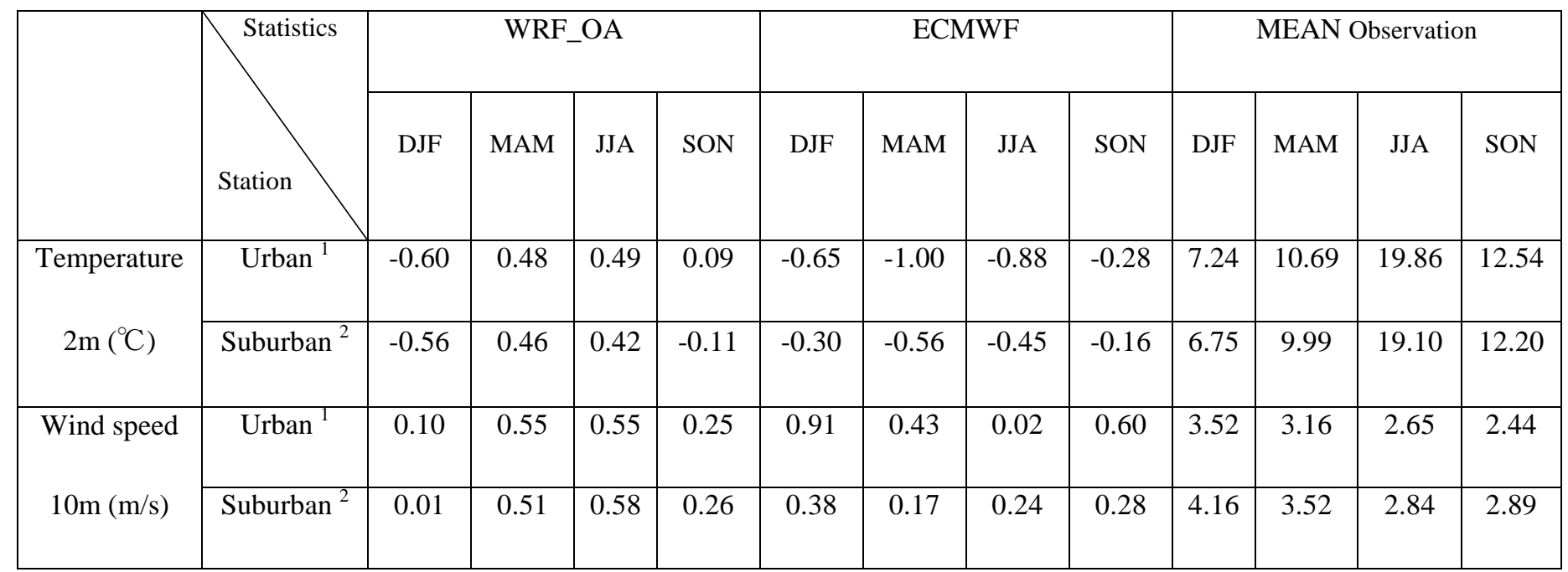

Groups of stations:

1 Montsouris, St-Maur, Orly, Longchamp

2 Roissy, Trappes, Villacoublay, Le Bourget

DJF: December-January-February (winter)

MAM: March-April-May (spring)

JJA: June-July-August (summer)

SON: September-October-November (autumn) 